\title{
Full Dose-Reduction Potential of Statistical Iterative Reconstruction for Head CT Protocols in a Predominantly Pediatric Population
}

\author{
(D) A.E. Mirro, (D) S.L. Brady, and (D)R.A. Kaufman
}

\begin{abstract}
BACKGROUND AND PURPOSE: A statistical iterative reconstruction algorithm provides an effective approach to reduce patient dose by compensating for increased image noise in CT due to reduced radiation output. However, after a point, the degree to which a statistical iterative algorithm is used for image reconstruction changes the image appearance. Our aim was to determine the maximum level of statistical iterative reconstruction that can be used to establish dose-reduced head CT protocols in a primarily pediatric population while maintaining similar appearance and level of image noise in the reconstructed image.
\end{abstract}

MATERIALS AND METHODS: Select head examinations (brain, orbits, sinus, maxilla, and temporal bones) were investigated. Dosereduced head protocols using an adaptive statistical iterative reconstruction were compared for image quality with the original filtered back-projection reconstructed protocols in a phantom by using the following metrics: image noise frequency (change in perceived appearance of noise texture), image noise magnitude, contrast-to-noise ratio, and spatial resolution. Dose-reduction estimates were based on CT dose index values. Patient volume CT dose index and image noise magnitude were assessed in 737 pre- and post-dose-reduced examinations.

RESULTS: Image noise texture was acceptable for up to $60 \%$ adaptive statistical iterative reconstruction for the soft reconstruction kernel (at both 100 and $120 \mathrm{kV}[$ peak]) and up to $40 \%$ adaptive statistical iterative reconstruction for the standard reconstruction kernel. Implementation of $40 \%$ and $60 \%$ adaptive statistical iterative reconstruction led to an average reduction in the volume CT dose index of $43 \%$ for brain, $41 \%$ for orbit, $30 \%$ for maxilla, $43 \%$ for sinus, and $42 \%$ for temporal bone protocols for patients between 1 month and 26 years of age, while maintaining an average noise magnitude difference of $0.1 \%$ (range, $-3 \%$ to $5 \%$ ), improving the contrast-to-noise ratio of low-contrast soft-tissue targets and the spatial resolution of high-contrast bony anatomy, compared with filtered back-projection.

CONCLUSIONS: The methodology in this study demonstrates maximizing patient dose reduction and maintaining image quality by using statistical iterative reconstruction for a primarily pediatric population undergoing head CT examinations.

ABBREVIATIONS: ASIR = adaptive statistical iterative reconstruction; CNR = contrast-to-noise ratio; CTDI $_{\text {vol }}=$ volume $\mathrm{CT}$ dose index; $\mathrm{FBP}=$ filtered backprojection; IR = iterative reconstruction; MTF = modulation transfer function; NPS = noise power spectrum

U se of statistical iterative reconstruction (IR) has been demonstrated as an effective method for lowering radiation exposure in thoracic and abdominal-pelvic CT. ${ }^{1-8}$ Recently, several studies have investigated a reduced dose in head CT by using statistical $\mathrm{IR}^{9-14}$; however, only 2 studies examined a pediatric

Received October 6, 2015; accepted after revision January 5, 2016

From the Department of Biomedical Engineering (A.E.M.), Washington University, St. Louis, Missouri; and Department of Diagnostic Imaging (A.E.M. S.L.B., R.A.K.), St Jude Children's Research Hospital, Memphis, Tennessee.

This work was supported by American Lebanese Syrian Associated Charities and National Cancer Institute R25E grant 5R25CA23944.

Please address correspondence to Samuel Brady, PhD, Department of Diagnostic Imaging, St Jude Children's Research Hospital, 262 Danny Thomas Pl, Memphis, TN 38105; e-mail: samuel.brady@stjude.org

- Indicates open access to non-subscribers at www.ajnr.org

http://dx.doi.org/10.3174/ajnr.A4754 population. ${ }^{10,11}$ These studies investigated the effect of statistical IR on image quality by using metrics such as noise magnitude, by measuring the interpixel variation or SD within an ROI. Measuring noise magnitude is simple but does not fully describe the effect that statistical IR algorithms have on the texture or the appearance of the pixelated noise, as has been reported previously. ${ }^{2,15,16}$

Current St Jude Children's Research Hospital examinations for the chest and abdomen-pelvis are performed on a LightSpeed VCT-XTe (GE Healthcare, Milwaukee, Wisconsin) and incorporate adaptive statistical iterative reconstruction (ASIR; GE Healthcare), ${ }^{1,2}$ but protocols involving the head (brain, orbits, sinus, maxilla, and temporal bone) are reconstructed by using filtered back-projection (FBP). The purpose of this study was to implement the maximum level of statistical IR for dose-reduced head protocols by using ASIR in a primarily pediatric population while maintaining a similar image-noise magnitude. Fourier- 
Table 1: Head CT examination parameters ${ }^{\mathrm{a}}$

\begin{tabular}{|c|c|c|c|c|c|c|c|c|}
\hline $\begin{array}{c}\text { Patient Age } \\
\text { (yr) }\end{array}$ & $\mathrm{A}$ or $\mathrm{H}$ & $\begin{array}{c}\text { Rotation } \\
\text { (sec) }\end{array}$ & $\begin{array}{l}\text { Collimation } \\
(\mathrm{mm})\end{array}$ & $\begin{array}{c}\text { Section } \\
(\mathrm{mm})\end{array}$ & $\begin{array}{c}\text { Reconstruction } \\
\text { Kernel }\end{array}$ & $k V p$ & $\begin{array}{l}\text { Pre-ASIR } \\
(\mathrm{mA})\end{array}$ & $\begin{array}{c}\text { Post-ASIR } \\
(\mathrm{mA})\end{array}$ \\
\hline \multicolumn{9}{|l|}{ Brain } \\
\hline $0-2^{b}$ & A & 0.5 & 20 & 5 & Soft and bone & 100 & 280 & 150 \\
\hline $2-5$ & & 1 & & & & & 200 & 120 \\
\hline $6-10$ & & & & & & & 220 & 130 \\
\hline 11-18 & & & & & & & 240 & 140 \\
\hline$\geq 19$ & & & & & & 120 & 200 & 105 \\
\hline \multicolumn{9}{|l|}{ Sinus } \\
\hline$\geq 19$ & $\mathrm{H}$ & 0.5 & 40 & 2.5 & Soft and bone & 120 & $\mathrm{NI}=7.5$ & 155 \\
\hline 0-18 & & & & & & 100 & 220 & 130 \\
\hline \multicolumn{9}{|l|}{ Orbits } \\
\hline $0-18^{c}$ & $\mathrm{H}^{\mathrm{d}}$ & 0.5 & 20 & 1.25 & Standard and bone & 100 & 240 & 155 \\
\hline \multicolumn{9}{|l|}{ Temporal bone } \\
\hline$\geq 19$ & $\mathrm{H}$ & 1 & 20 & 1.25 & Standard and bone & 120 & 250 & 150 \\
\hline $2-18$ & & 0.5 & & & & 120 & 400 & 230 \\
\hline \multicolumn{9}{|l|}{ Maxillary bone } \\
\hline$\geq 19$ & $\mathrm{H}^{\mathrm{d}}$ & 0.5 & 20 & 2.5 & Standard and bone & 120 & $\mathrm{NI}=7.5$ & $\mathrm{NI}=9.25$ \\
\hline 0-18 & $\mathrm{H}$ & & & & & 100 & 300 & 180 \\
\hline
\end{tabular}

Note:-A indicates axial; $\mathrm{H}$, helical; NI, Noise Index; SFOV, scan FOV.

a All protocols were imaged with a SFOV using "Head" unless otherwise indicated. All helical acquisitions were scanned with a pitch of 0.984 unless otherwise indicated.

b SFOV used "Ped Head."

c SFOV used "Small Head."

${ }^{\mathrm{d}}$ Pitch $=0.516$.

based image quality metrics, such as noise power spectrum (NPS) and modulation transfer function (MTF), were used to fully characterize the effects of ASIR on noise and spatial resolution. Dosereduction estimates are based on a comparison of pre- and postdose-reduced examination volume CT dose index $\left(\mathrm{CTDI}_{\mathrm{vol}}\right)$ values.

\section{MATERIALS AND METHODS}

\section{Head CT Image Quality Analyzed in a Phantom}

To determine the maximum possible level of statistical IR and tube current (ie, milliampere) reduction, we analyzed image quality from an ASIR reconstruction and compared it with image quality from the original head protocols by using FBP. Image quality was assessed in a phantom on the basis of the measured change of image noise frequency (ie, change in the perceived appearance of noise texture as quantified by calculating the NPS), image noise magnitude (ie, calculated by using the SD of an ROI), contrast-to-noise ratio (CNR), and spatial resolution (calculated by using MTF).

The NPS was calculated by using a 20 -cm diameter uniform water phantom (Quality Assurance Phantom; GE Healthcare). The water phantom was scanned to produce twelve $2.5-\mathrm{mm}$ images by using tube potential (ie, kilovolt[peak]) and other acquisition factors from the original head protocols (Table 1). The images were averaged, and the center of the averaged image was used to calculate a single NPS curve. ${ }^{2}$ Initially, the uniform water phantom was imaged at the $\mathrm{CTDI}_{\mathrm{vol}}$ and milliampere or, in the case of tube current-modulated examinations, the Noise Index value recorded for the original clinical FBP protocol. To produce a series of noisier images, we decremented the milliampere setting in steps of 10 milliamperes until the original CTDI $_{\text {vol }}$ was decreased by $\sim 70 \%$ (eg, for the brain protocol for individuals older than 19 years of age, the initial CTDI $_{\mathrm{vol}}$ and milliampere was 36.6 $\mathrm{mGy}$ and $200 \mathrm{~mA}$; both were decremented to $10.04 \mathrm{mGy}$ and 60 $\mathrm{mA}$ ); for head scan techniques imaged by using tube current modulation, the Noise Index value was incremented ${ }^{2}$ (thus allowing a lower milliampere) in steps of 3 . All other acquisition parameters were held constant (Table 1). Each milliampere-reduced image was reconstructed by using the soft, standard, and bone reconstruction kernels at every level of ASIR $(0 \%-100 \%$, in which $0 \%$ ASIR represents $100 \%$ FBP). Image noise magnitude, variance, and NPS were calculated by using a script written in Matlab (R2014b; MathWorks, Natick, Massachusetts).

The NPS of dose-reduced statistical IR data was grouped according to similar amplitudes (ie, the measure of noise variance) by reconstruction kernel type (ie, soft, standard, or bone), and kilovolt peak level (ie, 100 and 120). From these matched NPS curves, the shift in mean NPS frequency was calculated at each level of ASIR reconstruction. The texture of the image noise as it appeared in reconstructed images changed as the mean of the NPS curve shifted along the abscissa; thus, shifts in mean NPS frequency were associated with changes in image-noise texture (Fig 1) as has been shown in previous studies. ${ }^{2,16}$

A literature search was conducted to determine the level of acceptable shift in mean NPS frequency in lieu of a receiver operating characteristic test performed by radiologists at St Jude Children's Research Hospital. Acceptable changes in perceived noise texture determined by a single institutional receiver operating characteristic would not be generalizable, whereas a literature search represented a multi-institutional consensus. The resulting literature search indicated for soft-tissue reconstruction kernels, typical of body imaging (ie, the standard kernel), an average implementation of $40 \%$ ASIR reconstruction, ${ }^{1-3,6-8,17-19}$ correlated with an acceptable change in perceived image noise texture or mean NPS frequency shift of 25\% (range, 16\%-40\%). 2,4,5,20,21 No level of acceptable shift in mean NPS frequency was reported for the soft reconstruction kernel typical for brain CT. The tolerance of $25 \%$ reported for the standard reconstruction kernel was adopted for the soft reconstruction kernel. 

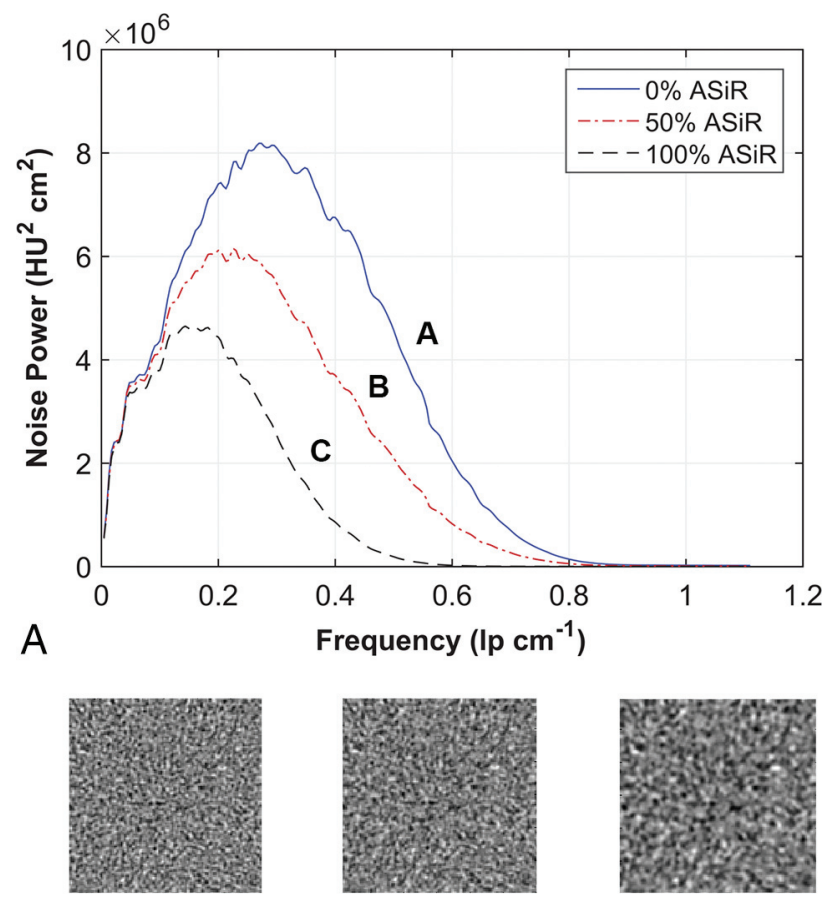

B Curve-A

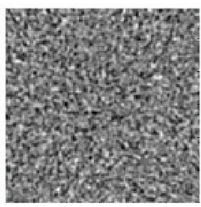

Curve-B

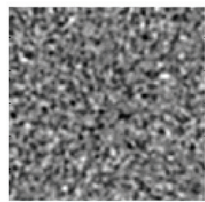

Curve-C

FIG 1. Texture of image noise as it appears in reconstructed images changes as the mean of the NPS curve shifts along the abscissa; shifts in mean NPS frequency are associated with changes in the appearance of image noise texture. $A$, NPS curves of the standard reconstruction kernel are reconstructed at 3 levels of ASIR. B, A corresponding ROI of $128 \times 128$ pixels from the center of a water phantom shows the appearance of the noise texture as it correlates with a 32\% shift in NPS mean frequency along the abscissa from curve $A$ to $B$ and a $52 \%$ shift in curve $A$ to $C$.

Images of low-contrast targets were acquired to qualitatively compare noise texture. The low contrast targets were imaged at multiple milliampere-reduced, ASIR-reconstructed levels and were compared with the original full-dose protocol by using FBP. Images of low-contrast targets were acquired with the soft and standard reconstruction kernels by using a Catphan 700 phantom (The Phantom Laboratory, Salem, New York), and the CNR of the 3-mm-diameter target was calculated. Additionally, a qualitative assessment of a low-contrast target was performed on a diagnostic-quality display (Dome S3c; NDS Surgical Imaging, San Jose, California) under reading room ambient light control (ie, illuminance average $\sim 20 \mathrm{~lx}$ ).

Fine-detailed spatial resolution was evaluated for the bone reconstruction kernel by calculating the MTF from images of highcontrast targets by using the Catphan 700 phantom. FBP and milliampere-reduced statistical IR images were used to image the phantom. Twelve scans of the first test module were acquired and averaged. The Fourier transform of the derivative of an ensemble of 1D edge spread functions sampled radially across the bone circular boundary insert was used to calculate the MTF. ${ }^{22}$

The percentage difference between milliampere values from the FBP image and the matched NPS curve reconstructed with statistical IR was used to determine new dose-reduced milliampere settings for all head protocols. All changes to protocols were reviewed by the chief neuroradiologist before implementation.
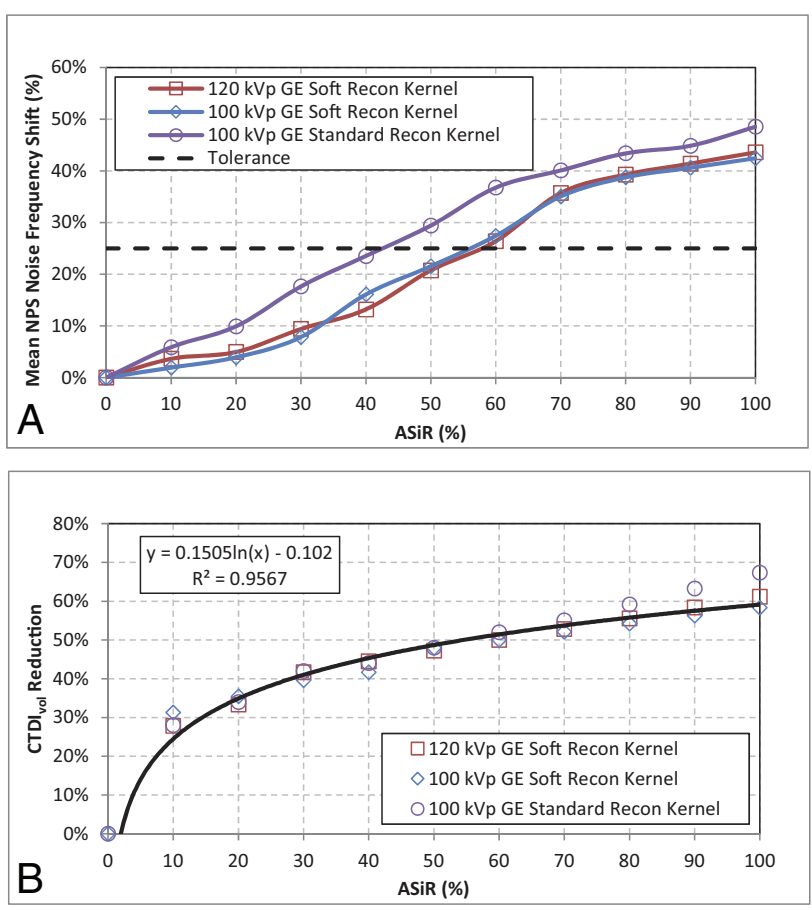

FIG 2. Dose-reduced ASIR protocols compare the mean NPS frequency shift $(A)$ as a function of the level of ASIR reconstruction. An acceptable tolerance for the appearance of noise texture in the reconstructed image is reported in the literature, ${ }^{4,5,20,21}$ based on a $25 \%$ shift of NPS noise frequency (dashed line). B, Corresponding reductions of CTDI ${ }_{\text {vol }}$ for the protocols by using ASIR are plotted and fit by using a log-regression function.

\section{Image Quality and Dosimetry Analyzed from Patient Examinations}

The institutional review board at St Jude Children's Research Hospital deemed this quality-assurance analysis exempt from obtaining informed consent. All data were managed in compliance with the Health Insurance Portability and Accountability Act. Head protocols were selected on the basis of each patient's age, which was obtained immediately prior to the examination. Predose-reduced examination $\mathrm{CTDI}_{\mathrm{vol}}$ values were analyzed from June 2013 to 2014. Post-dose-reduced values were analyzed from June 2014 to 2015.

Reconstructed image-noise magnitude from pre- and postdose-reduced patient examination images was assessed on the basis of an ROI analysis. Multiple ROIs were placed in regions of uniformity within the brain and averaged; the locations varied depending on the examination type. Image noise analysis was only for images reconstructed with soft-tissue reconstruction kernels (ie, soft or standard).

\section{RESULTS}

\section{Head CT Image Quality Analyzed in a Phantom}

Eleven NPSs (1 FBP, 10 ASIR spectra) were calculated for the soft reconstruction kernel (at both 100 and $120 \mathrm{kVp}$ ) and the standard reconstruction kernel $(100 \mathrm{kVp})$. The percentage shift in mean NPS frequency for each spectrum was plotted as a function of the level of ASIR (Fig 2A) with its accompanying reduction in $\mathrm{CTDI}_{\mathrm{vol}}$ (Fig 2B). The shift of mean NPS frequency (ie, noise texture) was impacted mostly by selection of the reconstruction kernel, and not the level of kilovolt (peak). On the basis of the reported ${ }^{4,5,20,21}$ 
25\% threshold for acceptable change in perceived noise texture (dashed line in Fig 2A), an implementation of 60\% ASIR was chosen for the soft reconstruction kernel and $40 \%$ ASIR was chosen for the standard reconstruction kernel; the data for the standard reconstruction kernel agree with those in previous publications. ${ }^{3,6-8,17-19}$ Dosereduced NPS curves for the soft reconstruction kernel were calculated for up to $60 \%$ ASIR and, for the standard reconstruction kernel, up to $40 \%$ ASIR (Fig 3). The overall noise magnitude and variance for the dose-reduced ASIR spectra were matched to the original FBP noise amplitude to a mean $( \pm 1 \mathrm{SD})$ of $4.8 \pm 0.4 \mathrm{HU}, 4.1 \pm 0.3 \mathrm{HU}$, and $5.7 \pm 0.5 \mathrm{HU}$ for protocols acquired with the soft reconstruction kernel at $100 \mathrm{kVp}$ (Fig 3A) and $120 \mathrm{kVp}$ (Fig 3B) and the standard reconstruction kernel at $100 \mathrm{kVp}$ (Fig $3 C$ ), respectively.

The visual assessment of low-contrast targets demonstrates a slight degradation in lesion boundary sharpness with the ASIR reconstruction (Fig 4). However, for images reconstructed with the soft reconstruction kernel, CNR improved with the increasing level of ASIR reconstruction. The smallest low-contrast target (3 $\mathrm{mm}$ ) acquired at $100 \mathrm{kVp}$ had a CNR calculated to be 1.2 at $0 \%$ ASIR and 2.4 at $60 \%$ ASIR. For targets acquired at $120 \mathrm{kVp}$, the CNR was calculated to be 1.7 at $0 \%$ ASIR and 2.3 at $60 \%$ ASIR. For targets acquired with the standard reconstruction kernel, the CNR improved up to the level of 30\% ASIR; the CNR was 1.4 at $0 \%$ ASIR and 1.9 at 30\% ASIR; however, the CNR was only 1.8 at $40 \%$ ASIR, a slight decrease from $30 \%$ ASIR.

For image-quality measurements of the bone reconstruction kernel, the dose-reduced NPS demonstrated an overall average reduction in noise variance by $26 \%$ (range, 7\%-36\%) compared with non-dose-reduced FBP protocols. Additionally, spatial resolution calculated for the dose-reduced $60 \%$ ASIR protocol improved by an average of $26 \%$ (range, $24 \%-30 \%$ ) compared at the $50 \%$ MTF level and $113 \%$ (range, 101\%-123\%) at the 10\% MTF level.

\section{Image Quality and Dosimetry Analyzed from Patient Examinations}

The total number of pre-dose-reduced examinations analyzed was 376 ( 242 male); the mean age was $9.6 \pm 6.2$ years ( 1 month to 24 years). The number of examinations analyzed per protocol was the following: 220 brain, 11 orbit, 98 sinus, 37 maxilla, and 10 temporal bone examinations. The total number of post-dose-reduced examinations analyzed was 361 (212 male); the mean age was $10.7 \pm 6.6$ years ( 1 month to 26 years). The number of examinations analyzed per protocol was the following: 193 brain, 3 orbit, 127 sinus, 35 maxilla, and 3 temporal bone examinations. Lowering the protocol milliampere (Table 1) and implementing $40 \%$ or $60 \%$ ASIR for image noise control resulted in lowered $\mathrm{CTDI}_{\mathrm{vol}}$ values as shown in Fig $3 \mathrm{~B}$. The percentage reduction in $\mathrm{CTDI}_{\mathrm{vol}}$ for all examinations is shown in Table 2. The image noise magnitude from the dose-reduced patient examinations was shown to change by an average difference of $0.1 \%$ (range, $-3 \%$ to $5 \%$ ) compared with the original FBP patient examinations (Table 2).

\section{DISCUSSION}

The purpose of this study was to implement the maximum level of statistical IR that could be used to establish dose-reduced pediatric head protocols (ie, brain, orbit, sinus, maxilla, and temporal
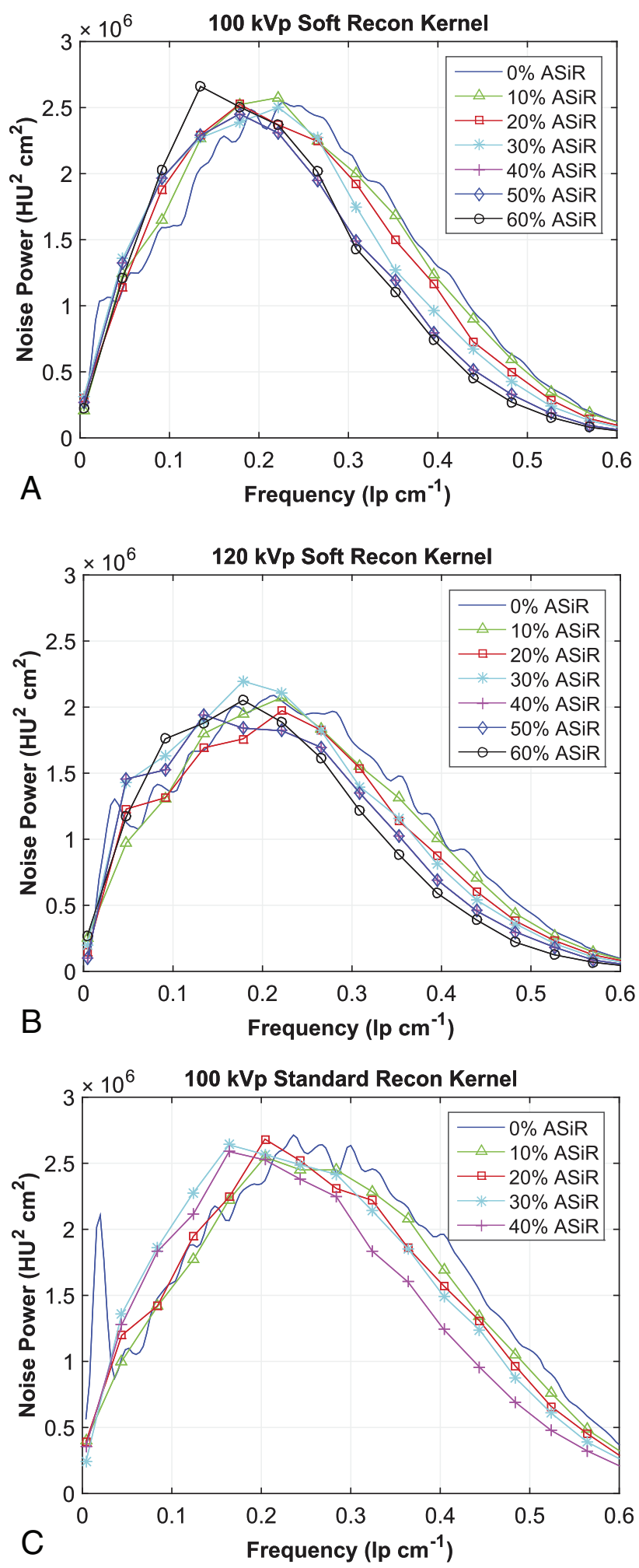

FIG 3. Noise power spectra acquired with the soft reconstruction kernel at $100 \mathrm{kVp}$ from 240 to $120 \mathrm{~mA}(A)$, with the soft reconstruction kernel at $120 \mathrm{kVp}$ from 200 to $110 \mathrm{~mA}(B)$, and the standard reconstruction kernel at $100 \mathrm{kVp}$ from 250 to $140 \mathrm{~mA}(C)$. The calculated spectra are reconstructed at 0\%-60\% ASIR (soft reconstruction kernel) and at $0 \%-40 \%$ ASIR (standard reconstruction kernel).

bone) while maintaining acceptable image quality. The use of NPS to evaluate image quality is a departure from the more commonly used metrics of CNR, SNR, and SD as previously reported. ${ }^{11-14}$ 
Using NPS allowed the definition of acceptable image quality to be based on the results from multiple published observer studies instead of a single-institute analysis; thus, the results of this analysis will be more generalizable across pediatric imaging institutions. The results of this study provide a more in-depth description of image appearance and noise texture and demonstrate a methodic approach for the application of the highest possible dose reduction by using statistical IR while maintaining a similar noise magnitude in the reconstructed image.

Images acquired with higher levels of statistical IR can appear overly smooth, leading to concerns about the visibility of anatomic structures. This change in image appearance is likely a visual manifestation of a shift in the spatial frequency distribution of the image noise. By measuring the mean frequency of the NPS curves, the image noise texture produced by ASIR for the dosereduced protocols could be compared with the image texture produced by the original FBP protocols, allowing the selection of acceptable change in noise texture. While the dose-reduced protocols did result in changes in the spatial frequency, these shifts were similar to the reported tolerance for soft-tissue imaging in
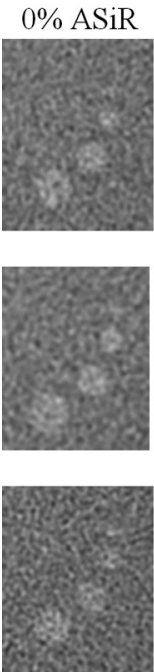

$10 \%$ ASiR
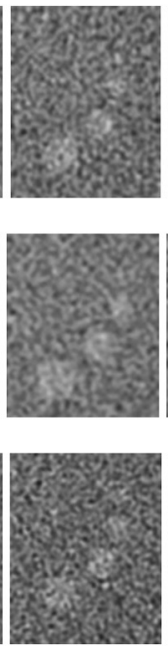
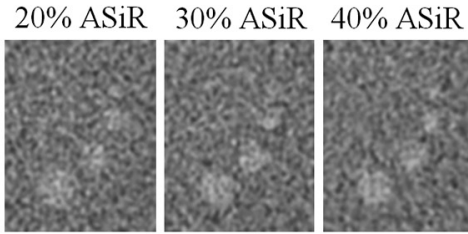

Soft Kernel 100 kVp

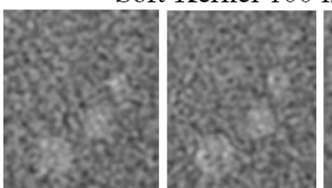

Soft Kernel $120 \mathrm{kVp}$

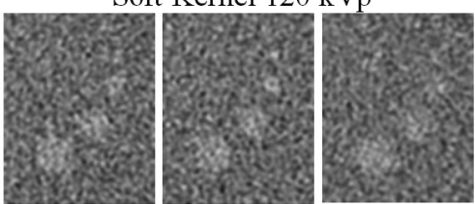

Standard Kernel 100 kVp
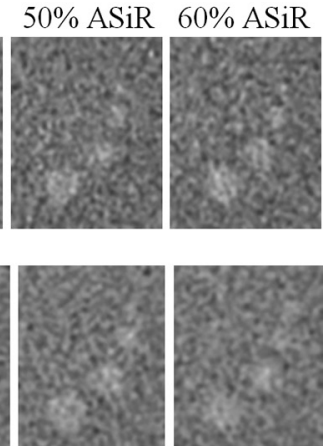

determined by the radiologists at our institution.

In 1 clinical example, image noise magnitude was measured in 2 axial brain examinations of a 16-kg (3-year-old) patient, performed approximately 6 months apart. The first scan (Fig 5A) was acquired with the original institutional protocol, and the second (Fig 5B), with the dose-reduced protocol at $60 \%$ ASIR. Noise texture appearance was slightly coarser, but the noise magnitude, as measured by the SD of a $1-\mathrm{cm}^{2} \mathrm{ROI}$, was $3.8 \mathrm{HU}$ in the preASIR image and $4.0 \mathrm{HU}$ in the post-ASIR image. The pre-ASIR image was acquired at $200 \mathrm{~mA}$, and the post-ASIR image, at 120 $\mathrm{mA}$ (both at $100 \mathrm{kVp}$ ); all other scan parameters were constant with a minor difference in gantry tilt angle to align with the orbitomeatal line. The change in milliampere represented a decrease in $\mathrm{CTDI}_{\mathrm{vol}}$ from 25.1 to $15.0 \mathrm{mGy}$, a dose reduction of $40 \%$.

A comparison of radiation dose reduction between FBP and dose-reduced ASIR brain protocols with previously published studies follows. By implementing 30\% ASIR reconstruction, Kilic et $\mathrm{al}^{11}$ reported a reduction of an adult brain protocol of $35 \%$ $\left(\mathrm{CTDI}_{\mathrm{vol}}, 59.4-38.6 \mathrm{mGy}\right)$, whereas the current study achieved a $48 \%$ dose reduction from 36.6 to 18.9 $\mathrm{mGy}$ in a population of patients 19 years of age or older, using $60 \%$ ASIR. For pediatric brain scans, Vorona et $\mathrm{al}^{9}$ reported a reduction of $22 \%\left(\mathrm{CTDI}_{\mathrm{vol}}\right.$, 28.8-22.4 mGy) for patients 3-18 years of age by using $20 \%$ ASIR, compared with the average reduction of $40 \%$ $\left(\mathrm{CTDI}_{\mathrm{vol}}, 26.5-15.8 \mathrm{mGy}\right)$ in the current study for the same age range by using $60 \%$ ASIR. Also, for pediatric brain scans, McKnight et al, ${ }^{10}$ using 30\% ASIR, reported a reduction of $\mathrm{CTDI}_{\mathrm{vol}}$ of 28\% (30.0-21.5 mGy) for patients 3-12 years of age and 48\% (49.9-25.7 $\mathrm{mGy}$ ) for patients older than 12 years of age, compared with the $40 \%$ (25.2-15.3 $\mathrm{mGy}$ ) and 45\% (32.9-18.0 mGy) dose reduction reported in the current study using 60\% ASIR, respectively. Percentage reductions are relative to the initial

F. Images of the 3-, 5-, 7-, and 9-mm low-contrast targets in the Catphan 700 phantom acquired with FBP and dose-reduced ASIR reconstruction up to $60 \%$ ASIR for the soft reconstruction kernels at both 100 and $120 \mathrm{kVp}$ and up to $40 \%$ for the standard reconstruction kernel at $100 \mathrm{kVp}$.

Table 2: Original and dose-reduced $\mathrm{CTDI}_{\mathrm{vol}}$ and noise values for all head protocols

\begin{tabular}{|c|c|c|c|c|c|c|c|}
\hline \multirow{2}{*}{$\begin{array}{c}\text { Patient Age } \\
\text { Category }\end{array}$} & \multirow[b]{2}{*}{ Protocol } & \multicolumn{3}{|c|}{$\mathrm{CTDI}_{\mathrm{vol}}(\mathrm{mGy})$} & \multicolumn{3}{|c|}{ Noise (HU) } \\
\hline & & Original & Dose-Reduced & Difference & Original & Dose-Reduced & Difference \\
\hline $0-23 \mathrm{mo}$ & Brain & $15.0 \pm 0.7$ & $8.0 \pm 0.4$ & $-47 \%$ & $4.4 \pm 1.0$ & $4.2 \pm 0.7$ & $-3 \%$ \\
\hline $2-5 y r$ & Brain & $24.1 \pm 0.9$ & $14.6 \pm 0.6$ & $-39 \%$ & $4.2 \pm 0.7$ & $4.1 \pm 0.7$ & $-3 \%$ \\
\hline $6-10 \mathrm{yr}$ & Brain & $26.3 \pm 1.3$ & $15.9 \pm 0.4$ & $-40 \%$ & $4.1 \pm 0.5$ & $4.2 \pm 0.6$ & $4 \%$ \\
\hline $11-18 \mathrm{yr}$ & Brain & $29.1 \pm 0.9$ & $17.0 \pm 0.5$ & $-42 \%$ & $4.4 \pm 0.6$ & $4.5 \pm 0.6$ & $3 \%$ \\
\hline$\geq 19 \mathrm{yr}$ & Brain & $36.6 \pm 0.8$ & $18.9 \pm 0.5$ & $-48 \%$ & $4.3 \pm 0.6$ & $4.4 \pm 0.4$ & $3 \%$ \\
\hline $0-18$ yr & Maxilla & $19.4 \pm 0.0$ & $11.5 \pm 0.1$ & $-41 \%$ & $11.2 \pm 2.8$ & $11.6 \pm 1.6$ & $3 \%$ \\
\hline$\geq 19 \mathrm{yr}$ & Maxilla & $22.8 \pm 0.0$ & $18.7 \pm 0.2$ & $-18 \%$ & $9.6 \pm 1.4$ & $9.2 \pm 1.7$ & $-3 \%$ \\
\hline $0-18 \mathrm{yr}$ & Orbits & $26.9 \pm 8.0$ & $15.8 \pm 0.5$ & $-41 \%$ & $7.5 \pm 1.2$ & $7.2 \pm 0.1$ & $-4 \%$ \\
\hline $0-18 \mathrm{yr}$ & Sinus & $13.1 \pm 0.0$ & $7.2 \pm 0.3$ & $-45 \%$ & $8.5 \pm 1.2$ & $8.9 \pm 1.1$ & $5 \%$ \\
\hline$\geq 19 \mathrm{yr}$ & Sinus & $22.8 \pm 0.0$ & $13.7 \pm 0.1$ & $-40 \%$ & $8.3 \pm 0.9$ & $8.2 \pm 0.6$ & $-1 \%$ \\
\hline $2-18 \mathrm{yr}$ & Temporal & $40.7 \pm 0.0$ & $22.8 \pm 0.0$ & $-44 \%$ & $9.3 \pm 1.4$ & $9.2 \pm 1.2$ & $-2 \%$ \\
\hline$\geq 19 \mathrm{yr}^{\mathrm{a}}$ & Temporal & $49.9 \pm 0.0$ & $29.7 \pm 0.0$ & $-40 \%$ & $9.3 \pm 1.0$ & & \\
\hline
\end{tabular}

${ }^{a}$ No dose-reduced patient examinations were available for comparison. Dose-reduced CTDI ${ }_{\text {vol }}$ value is calculated on the basis of scan parameters. Dose difference is a theoretic calculation. 


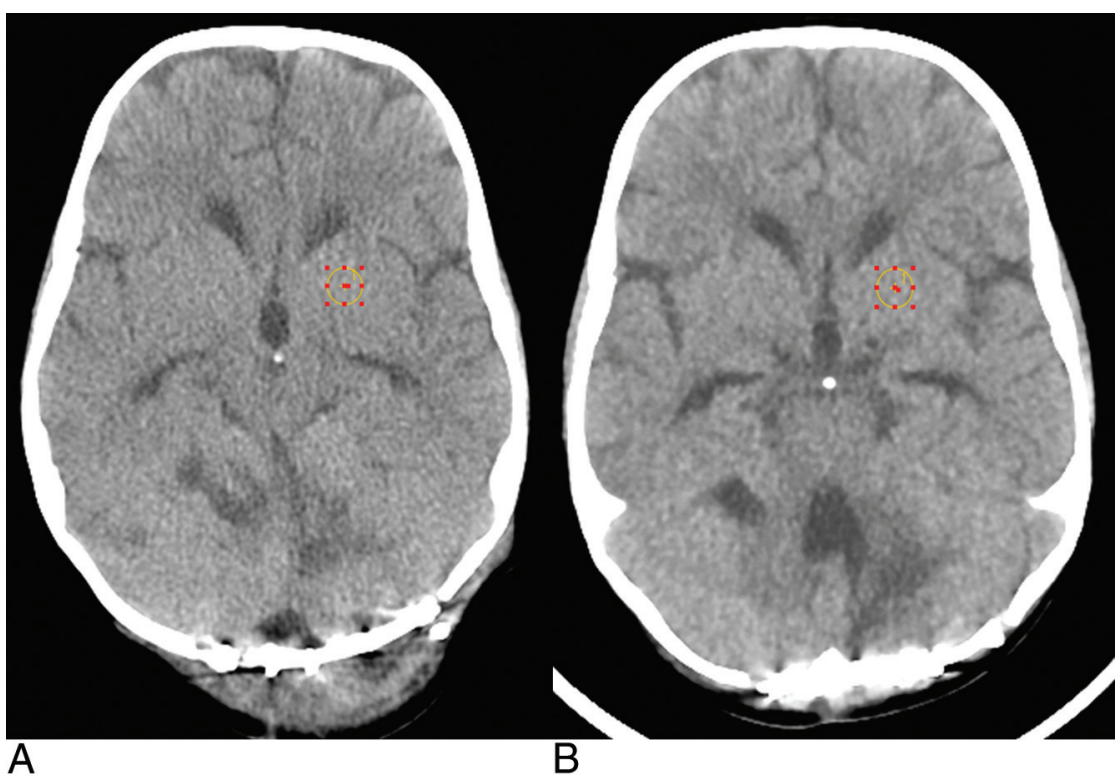

FIG 5. A 3-year-old boy with scans 6 months apart. $A$, The original brain protocol is acquired at $200 \mathrm{~mA}$ and $100 \mathrm{kVp}$ with a CTDI ${ }_{\mathrm{vol}}$ of $25.1 \mathrm{mGy}$ by using FBP. B. The patient is re-examined postsurgery with the dose-reduced brain protocol by using $60 \% \mathrm{ASIR}, 120 \mathrm{~mA}$, and $100 \mathrm{kVp}$, with $\mathrm{a} \mathrm{CTDI}_{\mathrm{vol}}$ of $15.0 \mathrm{mGy}$. Both examinations were acquired by using the GE Healthcare soft reconstruction kernel. noise texture in the reconstructed image as defined in the scientific literature and may be used for greater dose reduction. Head CT images acquired with the soft and standard reconstruction kernels demonstrated an overall improvement of CNR of the image. For all head protocols, the average reduction in $\mathrm{CTDI}_{\mathrm{vol}}$ was $43 \%$ for the brain, $41 \%$ for orbits, $30 \%$ for the maxilla, $43 \%$ for the sinus, and $42 \%$ for the temporal bone.

\section{ACKNOWLEDGMENTS}

The authors acknowledge Zoltan Patay, $\mathrm{MD}, \mathrm{PhD}$, for his advice and expertise.

Disclosures: Amy E. Mirro, Samuel L. Brady—RELATED: Grant: National Cancer Institute.* Robert A. Kaufman-RELATED: Grant: Pediatric Oncology Education students are supported in part by grant R25CA23944 from the National Cancer Institute, * Comments: This funds partial support of the summer student Pediatric Oncology Education program at St. Jude, which helped to support the coauthor Amy E. Mirro, an undergraduate student at Washington University in St. Louis. *Money paid to the institution.
$\mathrm{CTDI}_{\mathrm{vol}}$ calculated by using FBP reconstruction. Similarities in dose reduction between the current study and other previous studies, despite differences in the level of statistical IR implementation, are due to differences in the initial FBP CTDI ${ }_{\text {vol }}$ values.

In the current study, the statistical IR technique ASIR was used to mitigate increased image noise from the reductions of tube current, allowing reduced patient examination radiation dose. The use of ASIR is only available on GE Healthcare scanners. Other statistical IR algorithms are available for use with other CT manufacturers and may be used for potential head CT dose-reduction purposes. The implementation of these statistical IR algorithms will be subtly different; thus, the description of image noise texture and the amount of dose reduction reported in the current study may not be identical to those in other scanners using statistical IR algorithms for dose-reduced head CT. However, the principles outlined in the methodology of this study are universal, namely the need to analyze both image noise magnitude (ie, by using traditional ROI analysis) and the visual perception of the noise texture (ie, by using Fourier analysis techniques such as NPS) for a more complete understanding of the impact on reconstructed patient image quality from statistical IR. The use of Fourier image-quality metrics, such as NPS and MTF, will allow a more detailed analysis and customization of a statistical IR algorithm, despite the application.

\section{CONCLUSIONS}

Substantial dose reduction can be achieved at higher levels of ASIR reconstruction than previously reported for head CT protocols. An analysis of the effects on the perceived appearance of noise texture from implementation of statistical IR was performed. In this study, it was shown that an implementation of $60 \%$ ASIR (soft reconstruction kernel) and 40\% ASIR (standard reconstruction kernel) will produce acceptable changes in image

\section{REFERENCES}

1. Brady S, Moore B, Yee B, et al. Pediatric CT: implementation of ASIR for substantial radiation dose reduction while maintaining preASIR image noise. Radiology 2014;270:223-31 CrossRef Medline

2. Brady SL, Yee BS, Kaufman RA. Characterization of adaptive statistical iterative reconstruction algorithm for dose reduction in CT: a pediatric oncology perspective. Med Phys 2012;39:5520-31 CrossRef Medline

3. Hara AK, Paden RG, Silva AC, et al. Iterative reconstruction technique for reducing body radiation dose at CT: feasibility study. $A J R$ Am J Roentgenol 2009;193:764-71 CrossRef Medline

4. Hong SS, Lee JW, Seo JB, et al. Evaluation of image quality and radiation dose by adaptive statistical iterative reconstruction technique level for chest CT examination. Radiat Prot Dosimetry 2013; 157:163-71 CrossRef Medline

5. Sagara Y, Hara AK, Pavlicek W, et al. Abdominal CT: comparison of low-dose CT with adaptive statistical iterative reconstruction and routine-dose CT with filtered back projection in $\mathbf{5 3}$ patients. $A J R$ Am J Roentgenol 2010;195:713-19 CrossRef Medline

6. Singh S, Kalra MK, Gilman MD, et al. Adaptive statistical iterative reconstruction technique for radiation dose reduction in chest $\mathrm{CT}$ : a pilot study. Radiology 2011;259:565-73 CrossRef Medline

7. Singh S, Kalra MK, Hsieh J, et al. Abdominal CT: comparison of adaptive statistical iterative and filtered back projection reconstruction techniques. Radiology 2010;257:373-83 CrossRef Medline

8. Vorona GA, Ceschin RC, Clayton BL, et al. Reducing abdominal CT radiation dose with the adaptive statistical iterative reconstruction technique in children: a feasibility study. Pediatr Radiol 2011;41: 1174-82 CrossRef Medline

9. Vorona GA, Zuccoli G, Sutcavage $\mathrm{T}$, et al. The use of adaptive statistical iterative reconstruction in pediatric head CT: a feasibility study. AJNR Am J Neuroradiol 2013;34:205-11 CrossRef Medline

10. McKnight $C D$, Watcharotone $K$, Ibrahim $M$, et al. Adaptive statistical iterative reconstruction: reducing dose while preserving image quality in the pediatric head CT examination. Pediatr Radiol 2014; 44:997-1003 CrossRef Medline

11. Kilic K, Erbas G, Guryildirim M, et al. Lowering the dose in head CT using adaptive statistical iterative reconstruction. AJNR Am J Neuroradiol 2011;32:1578-82 CrossRef Medline 
12. Korn A, Fenchel M, Bender B, et al. Iterative reconstruction in head $\mathrm{CT}$ : image quality of routine and low-dose protocols in comparison with standard filtered back-projection. AJNR Am J Neuroradiol 2012;33:218-24 CrossRef Medline

13. Niu YT, Mehta D, Zhang ZR, et al. Radiation dose reduction in temporal bone $\mathrm{CT}$ with iterative reconstruction technique. AJNR Am J Neuroradiol 2012;33:1020-26 CrossRef Medline

14. Rapalino O, Kamalian S, Kamalian S, et al. Cranial CT with adaptive statistical iterative reconstruction: improved image quality with concomitant radiation dose reduction. AJNR Am J Neuroradiol 2012;33:609-15 CrossRef Medline

15. Samei E, Richard S. Assessment of the dose reduction potential of a model-based iterative reconstruction algorithm using a task-based performance metrology. Med Phys 2015;42:314-23 CrossRef Medline

16. Solomon J, Samei E. Quantum noise properties of CT images with anatomical textured backgrounds across reconstruction algorithms: FBP and SAFIRE. Med Phys 2014;41:091908 CrossRef Medline

17. Cornfeld D, Israel G, Detroy E, et al. Impact of adaptive statistical iterative reconstruction (ASIR) on radiation dose and image quality in aortic dissection studies: a qualitative and quantitative analysis. AJR Am J Roentgenol 2011;196:W336-40 CrossRef Medline
18. Flicek KT, Hara AK, Silva AC, et al. Reducing the radiation dose for CT colonography using adaptive statistical iterative reconstruction: a pilot study. AJR Am J Roentgenol 2010;195:126-31 CrossRef Medline

19. Leipsic J, Nguyen G, Brown J, et al. A prospective evaluation of dose reduction and image quality in chest $\mathrm{CT}$ using adaptive statistical iterative reconstruction. AJR Am J Roentgenol 2010;195:1095-99 CrossRef Medline

20. Marin D, Nelson RC, Schindera ST, et al. Low-tube-voltage, hightube-current multidetector abdominal CT: improved image quality and decreased radiation dose with adaptive statistical iterative reconstruction algorithm-initial clinical experience. Radiology 2010;254:145-53 CrossRef Medline

21. Miéville FA, Gudinchet F, Brunelle F, et al. Iterative reconstruction methods in two different MDCT scanners: physical metrics and 4-alternative forced-choice detectability experiments-a phantom approach. Phys Med 2013;29:99-110 CrossRef Medline

22. Richard S, Husarik DB, Yadava G, et al. Towards task-based assessment of CT performance: system and object MTF across different reconstruction algorithms. Med Phys 2012;39:4115-22 CrossRef Medline 